\title{
Anomalous and anisotropic nonlinear susceptibility in the proximate Kitaev magnet $\alpha-\mathrm{RuCl}_{3}$
}

\author{
Ludwig Holleis ${ }^{1}$, Joseph C. Prestigiacomo ${ }^{2}$, Zhijie Fan (D)', Satoshi Nishimoto $\mathbb{D}^{3,4}$, Michael Osofsky ${ }^{2}$, Gia-Wei Chern ${ }^{1}$, \\ Jeroen van den Brink (iD) ${ }^{3,4}$ and B. S. Shivaram (iD)
}

The leading order nonlinear (NL) susceptibility, $X_{3}$, in a paramagnet is negative and diverges as $T \rightarrow 0$. This divergence is destroyed when spins correlate and the NL response provides unique insights into magnetic order. Dimensionality, exchange interaction, and preponderance of quantum effects all imprint their signatures in the NL magnetic response. Here, we study the NL susceptibilities in the proximate Kitaev magnet $a-\mathrm{RuCl}_{3}$, which differs from the expected antiferromagnetic behavior. For $T<T_{\mathrm{c}}=7.5 \mathrm{~K}$ and field $B$ in the $a b$-plane, we obtain contrasting NL responses in low $(<2 \mathrm{~T})$ and high field regions. For low fields, the NL behavior is dominated by a quadratic response (positive $X_{2}$ ), which shows a rapid rise below $T_{c}$. This large $X_{2}>0$ implies a broken sublattice symmetry of magnetic order at low temperatures. Classical Monte Carlo (CMC) simulations in the standard $K-H-\Gamma$ model secure such a quadratic $B$ dependence of $M$, only for $T \approx T_{c}$ with $X_{2}$ being zero as $T \rightarrow 0$. It is also zero for all temperatures in exact diagonalization calculations. On the other hand, we find an exclusive cubic term $\left(X_{3}\right)$ that describes the high field NL behavior well. $X_{3}$ is large and positive both below and above $T_{c}$ crossing zero only for $T>50 \mathrm{~K}$. In contrast, for $B \| c$-axis, no separate low/high field behaviors are measured and only a much smaller $X_{3}$ is apparent.

npj Quantum Materials (2021)6:66; https://doi.org/10.1038/s41535-021-00364-z

\section{INTRODUCTION}

Since the demonstration by Kitaev ${ }^{1}$ of the existence of a quantum spin liquid state in two dimensions through exact calculations on a honeycomb lattice, there has been an intense experimental search for its realization in real material systems. Aided through insights provided by Jackeli and Khaliullin ${ }^{2}$ to engineer (bonddependent) exchange interactions, the materials search has identified several promising systems with prominent attention given thus far to two candidates, the iridium oxides and ruthenium chloride ${ }^{3,4}$. Despite the presence of a very large exchange energy (of the order of $100 \mathrm{~K}$ ), these systems do not order down to comparatively low temperatures. When they do order magnetically, the features observed in both microscopic probes such as neutron scattering and macroscopic measurements such as magnetometry and thermal response are highly unusual and contrast dramatically with what is expected from conventional magnets. In their thermal transport ${ }^{5}$, thermodynamic $^{6}$ and microwave response ${ }^{7}$, they provide tell-tale signs as to the presence of fractionalized excitations sought after in Kitaev magnets.

In the proximate Kitaev spin liquid candidate, $a-\mathrm{RuCl}_{3}$ neutron scattering experiments show a low-temperature magnetic excitation spectrum consisting of sharp spin wave peaks and a continuum associated with fractional excitations $s^{6,8}$. A magnetic transition that sets in at $7.5 \mathrm{~K}$ where the spins assume a zig-zag chain pattern located in the $a b$-plane with two neighboring chains being antiferromagnetically aligned ${ }^{9}$ is also established. Raman scattering studies also reveal unconventional magnetic excitations with a broad continuum whose temperature dependence is apparent over a large scale compared to the magnetic ordering temperature ${ }^{10,11}$. The linear susceptibility shows a discontinuity in slope $^{12}$ at $T_{\mathrm{N}}=7.5 \mathrm{~K}$ and exhibits substantial in-plane anisotropy that persists in the normal state ${ }^{13}$. The high temperature $(T>$
$150 \mathrm{~K})$ susceptibility is convincingly Curie-like; however, there is an extended intermediate "Kitaev paramagnetic" region ${ }^{6}$ beyond $T_{\text {c }}$. The out-of-plane anisotropies are also significant: the susceptibility parallel to the $c$-axis is nearly an order of magnitude smaller with only a minor signature at the $7.5 \mathrm{~K}$ transition. These magnetic signatures are in stark contrast to what is known in conventional 2D (insulating) antiferromagnets ${ }^{14}$.

In this communication, we report measurements of the nonlinear DC susceptibilities, $X_{2}$ and $X_{3}$, in $a-\mathrm{RuCl}_{3}$ and illustrate that they probe many key aspects of the proximate Kitaev spin liquid state as well as the zig-zag antiferromagnetic phase. The equilibrium magnetization in any system can be written in the general form:

$M=X_{1} B+X_{2} B^{2}+X_{3} B^{3}+\cdots$

where the coefficients represent the various order susceptibilities. In particular, the coefficient $X_{2}$ is nonzero when time reversal symmetry is broken, while preserving lattice symmetry; for instance ${ }^{15}$, in ferromagnets.

The nonlinear susceptibility $X_{3}$ in a classical paramagnet is negative at all temperatures and diverges ${ }^{16}$ as $T \rightarrow 0$, while $X_{2}$ is nonexistent due to time-reversal symmetry. The negative divergence in $X_{3}$ can be interrupted, however, if the system develops long-range magnetic order. We illustrate in Fig. $1 \mathrm{~A}-\mathrm{C}$ the three known types of characteristic behavior of both the linear $\left(x_{1}\right)$ and the nonlinear $\left(X_{2}\right.$ and $\left.X_{3}\right)$ susceptibilities for typical ferromagnets, bipartite antiferromagnets, and spin glasses, respectively ${ }^{15,17-19}$. In all three cases, significant nonzero $x_{3}$ is found only in the vicinity of the critical temperature. It is worth noting that $X_{3}$ assumes only negative values at $T>T_{c}$ for all three types of magnets and tends to zero rather quickly as $T$ increases. The second-order susceptibility, $X_{2}$, is less studied and a nonzero $X_{2}$ is possible only when time-reversal symmetry is either explicitly or spontaneously

${ }^{1}$ Department of Physics, University of Virginia, Charlottesville, VA, USA. ${ }^{2}$ Naval Research Laboratory, Washington, DC, USA. ${ }^{3}$ Department of Physics, Technical University Dresden, Dresden, Germany. ${ }^{4}$ Institute for Theoretical Solid State Physics, IFW Dresden, Dresden, Germany. ${ }^{凶}$ email: bss2d@virginia.edu 


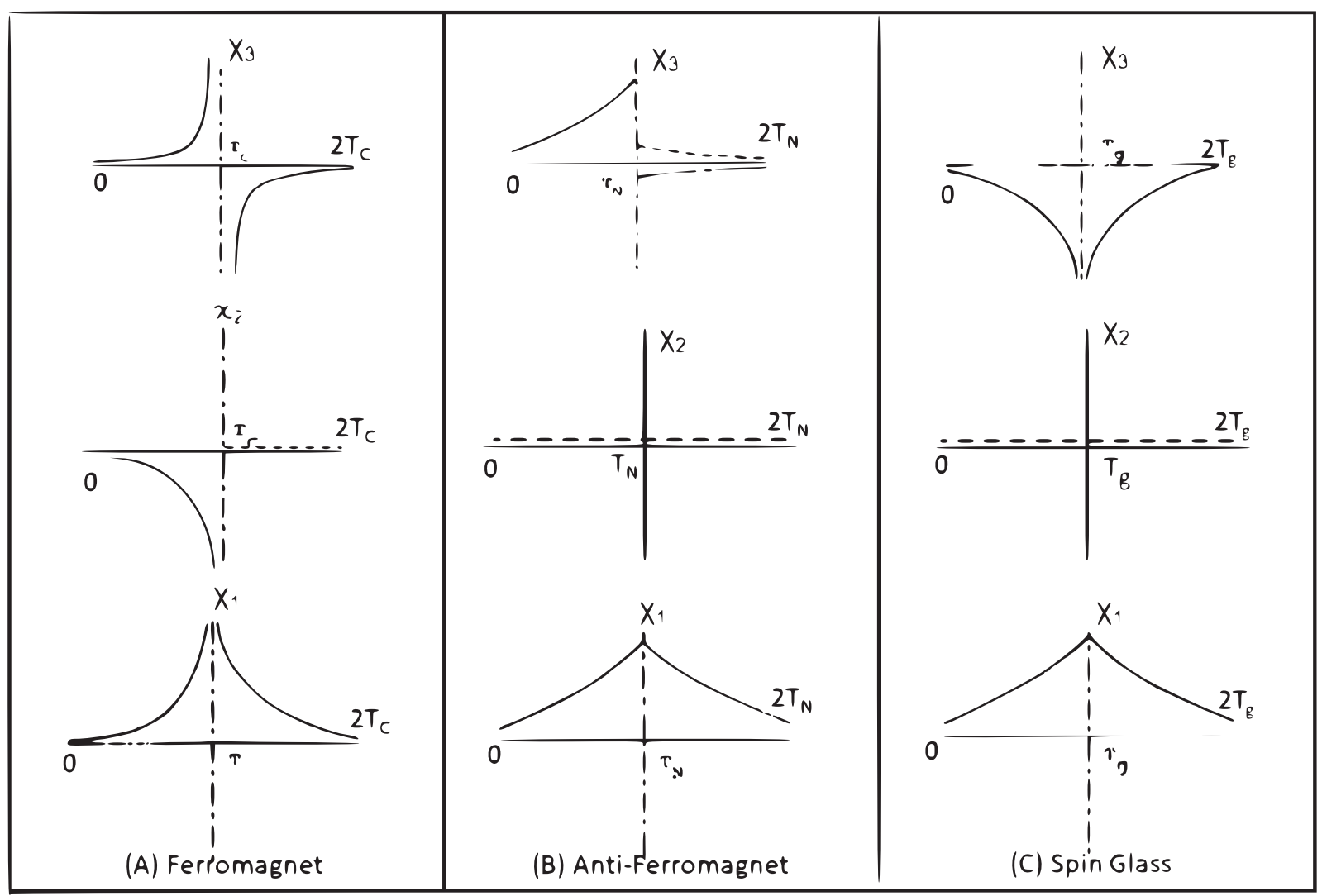

Fig. 1 Schematic of the known qualitative behaviors of the linear $\left(X_{1}\right)$ and nonlinear $\left(X_{2}\right.$ and $\left.X_{3}\right)$ magnetic susceptibilities in different flavors of magnetic systems. In the ferromagnet $(\mathbf{A})$ and spin glass $(\mathbf{C})$, the nonlinear susceptibility, $X_{3}$, is negative above the characteristic temperature and has a sharp discontinuity at a characteristic temperature $\left(T_{\mathrm{c}}\right.$ or $\left.T_{\mathrm{g}}\right)$. In the anti-ferromagnet (B), the sign of $X_{3}$ in the paramagnetic regime depends on the coordination number ${ }^{25}$. Below this temperature, it is observed to rapidly approach zero as $T \rightarrow 0$ for all three phases. $X_{2}$, on the other hand, exhibits such a discontinuity only for (A) (e.g., ferromagnet) where time-reversal symmetry is broken ${ }^{15}$. In comparison, in the other two cases it is zero.

broken, as in ferromagnets. Moreover, even for bipartite antiferromagnets, an effective time-reversal symmetry due to the sublattice symmetry results in a vanishing $X_{2}$ even in the ordered state below $T_{c}$; see Fig. 1B.

In $a-\mathrm{RuCl}_{3}$ we find, in contrast, that $\chi_{3}$ assumes significantly positive values over an extended temperature range above the ordering temperature. While there is a divergence of $X_{3}$ at $T_{\mathrm{c}}$ as might be expected, its value remains significantly positive down to the lowest temperatures. We also find a quadratic field dependence of $M$, giving rise to a significantly positive $X_{2}$, which exists only in the ordered state and has a large nonzero value even as $T \rightarrow 0$. To our knowledge, such a behavior of $X_{3}$ and $X_{2}$ have not been observed before. Further, this unexpected quadratic contribution is highly anisotropic and found only when the magnetic field is perpendicular to the high symmetry axis (c-axis).

\section{RESULTS AND DISCUSSION}

\section{Experimental results}

In Fig. 2, we show the measured magnetization isotherms for $B \|$ $a$-axis (i.e., $\phi=0^{\circ}$ as per the definition adopted in ref. ${ }^{13}$ ) plotted in a manner that facilitates the extraction of the nonlinear susceptibilities. Equation (1) above defines the susceptibility parameters and motivates plots such as Fig. 2. It is apparent from the top nine panels in Fig. 2 that the slope of the lines for $B \|$ $a$-axis, which are well defined and close to zero at high $T$, turn significantly positive when lowering $T$, and increase in magnitude as the ordering temperature $T_{\mathrm{c}}=7.5 \mathrm{~K}$ is approached. Below $T_{\mathrm{c}}$ the response breaks into two distinct regions, with a crossover threshold field of $B^{*} \approx 2 \mathrm{~T}$. The nonlinear response below $B^{*}$ is considerably larger than that at the high field end, $3 \mathrm{~T}<B<5 \mathrm{~T}$. Confining ourselves to the quadratic term only, as per Eq. (1) in this low field region, we obtain values of $X_{2}$ as shown in Fig. 3b. The values of $X_{2}$ are positive and large at the lowest temperatures and decrease monotonically towards $T_{\mathrm{c}}$, where it rapidly drops to zero. It is important to note that a nonzero $X_{2}$ is only possible in systems in which time-reversal symmetry is broken ${ }^{20}$. In a strict bipartite antiferromagnet, however, time-reversal symmetry is not broken. The fits described above also yield $X_{1}$ via the intercept, which can be used as a consistency check on the linear susceptibility values obtained at constant low field through temperature sweeps. The absence of any feature in $X_{1}$ in the vicinity of $14 \mathrm{~K}$ alludes to the high quality of the sample measured.

Another significant feature notable in the nine panels on the top in Fig. 2 is the presence of a clear upward curvature, in the 3-5 T region, particularly in the 6 and $10 \mathrm{~K}$ isotherms. This curvature implies the presence of a cubic term, which can be extracted by plotting $M / B$ against $B^{2}$ on the $x$-axis as shown in the bottom nine panels of Fig. 2. The values of $X_{3}$ obtained by fitting the linear region in such plots with $B$ between 3 and $5 T$ below $T_{c}$ and over the entire field range $(0-5 \mathrm{~T})$ above $T_{\mathrm{c}}$ are shown in Fig. $3 c$. The high linearity of these fits illustrates the absence of a $X_{2}$ term in these regions of the phase diagram. Performing such separate fits in the two regions is the most natural way to analyze our results. Our approach of separate fits is further motivated by 

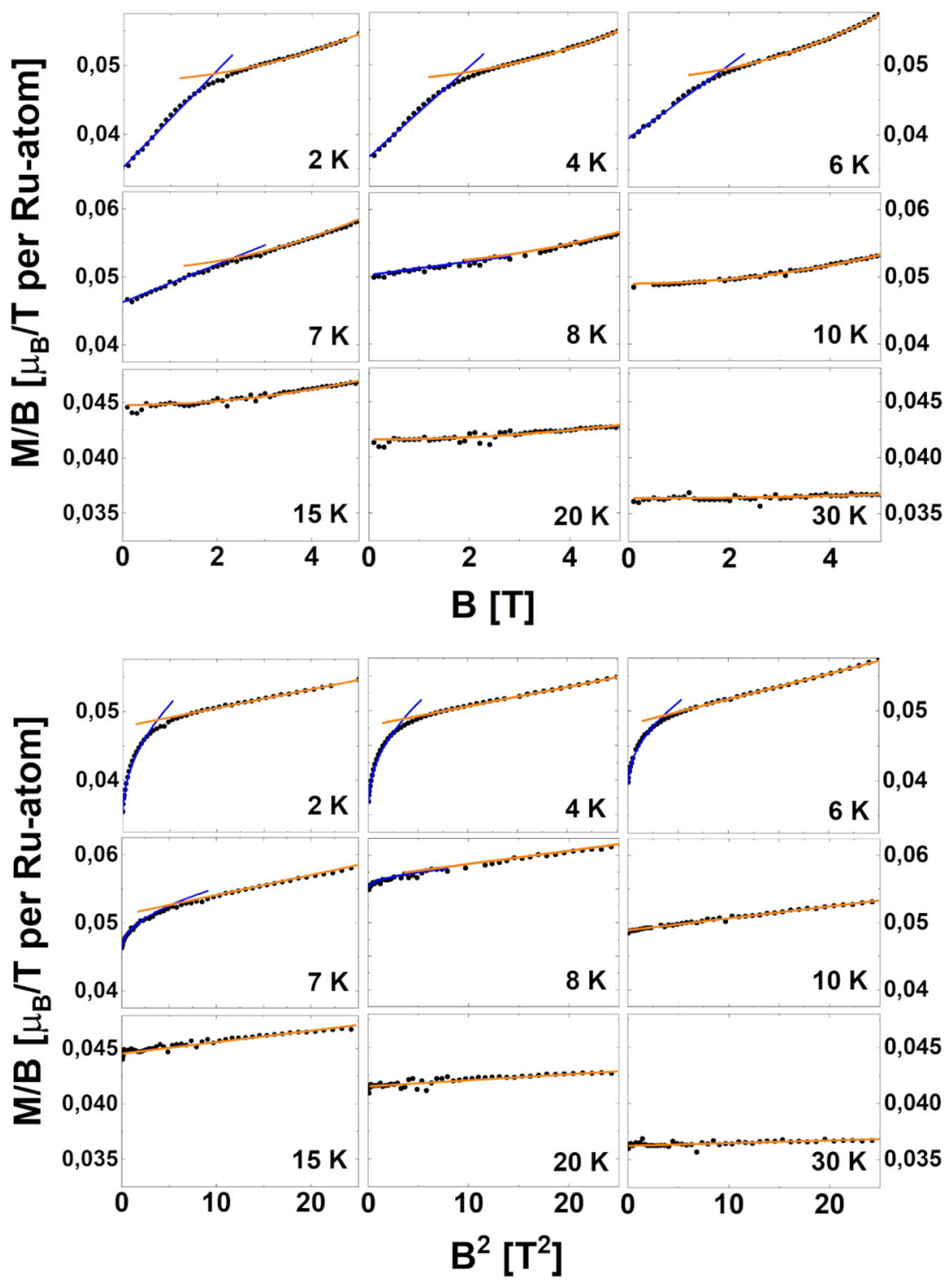

Fig. 2 Magnetization isotherms. The top set of nine panels show the ratio of the measured magnetization, $M$, to the magnetic field, $B$,

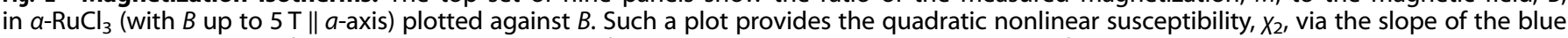
straight lines on the low field side. Since at the high field end $(B>3 T)$ the response is better fit with a cubic term $X_{3}$, we show in the nine panels on the bottom a similar plot but with $B^{2}$ on the abscissa and the fits in orange straight lines. It is clear from these panels that $X_{3}$ when $B \| a$-axis is positive over a wide temperature range, while $x_{2}$ vanishes for $T>T_{\mathrm{N}}$.

signs of a crossover transition at $\approx 2 \mathrm{~T}$ in neutron ${ }^{12,21}$, microwave response ${ }^{7}$, and differential susceptibility ${ }^{22}$ experiments.

As can be seen in Fig. $3 c_{1} X_{3}$ is significantly positive near $T_{c}$ and decreases in magnitude as the temperature is increased. A power-law fit as shown in the figure provides a reasonable empirical description of the behavior of $X_{3}$ in the (Kitaev) paramagnetic region. Experimentally, however, a crossover to negative $X_{3}$ occurs above temperature $50 \mathrm{~K}$ (a temperature of the order of the Kitaev exchange ${ }^{6}$ ). Below $T_{c}$ the values of the nonlinear susceptibility are obtained through linear fits in two separate regions as explained above. The high field region yields values for $X_{3}$ that exhibit a sharp peak at $T=6.5 \mathrm{~K}$ just below the ordering temperature. They remain positive down to the lowest temperature of $2 \mathrm{~K}$ with no evidence of a downturn towards zero. While a negative cubic power-law dependence is expected from the Curie law for the third-order susceptibility in paramagnets ${ }^{16}$, such a positive behavior has not been reported to our knowledge.
We have also measured the nonlinear susceptibility for magnetic fields perpendicular to the $a b$-plane. Representative magnetization isotherms plotted similar to those in Fig. 2 are shown in Fig. S2. The extracted values of $X_{3}$ are small and positive and increase monotonically as the temperature is lowered as seen in Fig. S3. More importantly, as evident in Fig. S2, the nonlinear response is well described by a single cubic term and there is no evidence for a large quadratic response as found for $B \| a$-axis. Given that for $B \| a$-axis the deviation from a linear behavior of the magnetization due to a quadratic contribution is roughly ten times larger than that arising from the cubic term, such behavior if present for $B \| c$-axis would be easily seen within the resolution of the MPMS SQUID measurements. We also present in Fig. S4 preliminary data for the in-plane nonlinear response when $B \perp a$-axis. While the behavior observed is qualitatively the same as in Fig. 3 , there are quantitative differences. Further characterization of this in-plane anisotropy will form part of a separate comprehensive study. 

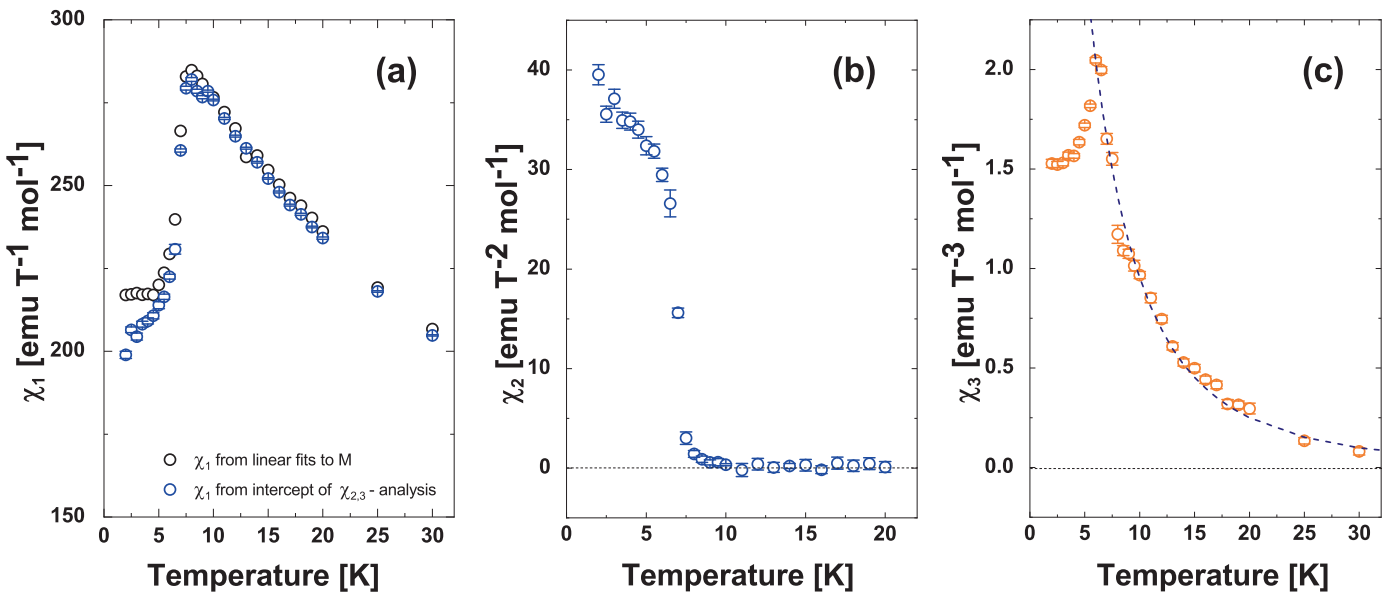

Fig. 3 Linear and higher-order susceptibilities. Panel (a) shows the linear susceptibility obtained through temperature sweep (black circles) as well as those obtained from the intercepts of the magnetization isotherms of the $\chi_{2}$ and $\chi_{3}$ analysis such as in Fig. 2 (blue circles). Panel (b) shows $X_{2}$ extracted from the low field quadratic response, from plots such as in Fig. 2, top nine panels. Similarly, panel (c) shows the temperature dependence of $X_{3}$ obtained from plots such as those in Fig. 2, bottom nine panels. A sharp "lambda"-like anomaly is apparent for $X_{3}$ obtained from the high field (3-5 T) response when $B \| a$-axis. The error bars capture the coefficient of determination of the linear fits such as shown in Fig. 2.

\section{Comparison to traditional nonlinear susceptibility responses}

Measurements of the equilibrium third-order susceptibility, although rare, have been performed in 2D magnets, frustrated systems, spin glasses, and strongly correlated itinerant metamagnets, that is, materials that show a rapid rise of magnetization at a critical field $B_{c}$ (see, for example, ref. ${ }^{23}$ ). In a bipartite antiferromagnet such as $\mathrm{FeCl}_{2}{ }^{24}$, or even in the classic 2D magnets, $\left(\mathrm{C}_{2} \mathrm{H}_{5} \mathrm{NH}_{3}\right)_{2} \mathrm{CuCl}_{4}{ }^{14}$, the DC nonlinear susceptibility, $X_{3}$, exhibits a "lambda"-like anomaly just below $T_{\mathrm{c}}$ as expected by the theory. From below $T_{c}$ the standard response of $X_{3}$ in these systems is a rapid rise to a large positive value at $T_{c}$, above which it drops with the sign being set by the coordination number ${ }^{25}$. For instance, in the paramagnetic phase of the Kagome system $\mathrm{SCGO}^{26}$, a large positive value of $\chi_{3}$ is seen above $T_{\mathrm{c}}$, but it reaches close to zero within $2 T_{\mathrm{c}}$. In many strongly correlated itinerant metamagnets ${ }^{27,28}$, in which an order parameter develops, $X_{3}$ peaks at the ordering temperature and decreases rapidly at $T \rightarrow 0$. Nonlinear susceptibility measurements in such systems typically probe higher-order correlations and place strong constraints on the ground state $e^{29,30}$. These latter systems are three-dimensional electronically, but can exhibit a strong magnetic anisotropy due to the $g$-factor. Nevertheless, in these systems, for all directions it is sufficient to include only the cubic term and the possibility of a dual response with a quadratic term has almost never been discussed (for an exception, see ref. ${ }^{31}$ ). Thus, the features reported above in $a-\mathrm{RuCl}_{3}$ are in contrast to much of what is known about nonlinear susceptibilities in magnets. The unmistakable presence of a large $T \rightarrow 0$ quadratic term makes $a-\mathrm{RuCl}_{3}$ a unique 2D quantum antiferromagnet.

\section{Comparison to simulations of the $\boldsymbol{J}_{1}-\boldsymbol{J}_{3}-\boldsymbol{K}-\Gamma$ model}

In order to understand the nature of the nonlinear susceptibility in $a-\mathrm{RuCl}_{3}$, it is possible to consider several approaches based on different model Hamiltonians employed thus $\operatorname{far}^{13,32}$. Most of these approaches start with the Kitaev-Heisenberg model appended with various choices of off-diagonal terms ${ }^{33,34}$. The correct choice of the Hamiltonian is still very much a matter of debate with the sign of the Kitaev term or even the necessity of the Kitaev term being in question ${ }^{35-37}$. We focus on this model and apply two separate calculation tools to study the nonlinear susceptibilities for $a-\mathrm{RuCl}_{3}$ : (a) we use CMC simulations with the model Hamiltonian

$$
\begin{aligned}
\mathcal{H}= & \sum_{\langle i j\rangle_{\gamma}}\left[J_{1} \mathbf{S}_{i} \cdot \mathbf{S}_{j}+K S_{i}^{\gamma} S_{j}^{\gamma}+\Gamma\left(S_{i}^{a} S_{j}^{\beta}+S_{i}^{\beta} S_{j}^{a}\right)\right] \\
& +\sum_{\langle\langle i j\rangle\rangle\rangle} J_{3} \mathbf{S}_{i} \cdot \mathbf{S}_{j}-g \mu_{B} \mathbf{B} \cdot \sum_{i} \mathbf{S}_{i}
\end{aligned}
$$

This so-called $J_{1}-J_{3}-K-\Gamma$ model is considered one of the the most successful efforts in modeling $a-\mathrm{RuCl}_{3}{ }^{32}$. (b) We employ exact diagonalization (ED) methods with slight adjustment on parameters as in ref. ${ }^{38}$. For (a), although quantum fluctuations are expected to be strong for such spin-1/2 systems, the fact that the magnet develops a long-range antiferromagnetic order justifies the classical spin approximation, as a first step to understanding the magnetic properties of the ordered phase. For (b), the choice is based on the recognition that it reproduces the magnetization isotherms to high fields very well.

With the CMC simulations we reproduce the phase transition to the so-called zig-zag order at $T_{\mathrm{c}} \approx 0.11 \mathrm{~K}$, where $K$ is the dominant Kitaev exchange constant, with the other parameter values normalized to $K$ taken as $\Gamma=0.5, J_{1}=0.036, J_{3}=0.035, g_{a}=g_{b}$ $=2.3$, and $g_{c}=1.3^{39,40}$. Our calculations of the linear susceptibility $X_{1}$ based on the Monte Carlo data, as shown in Fig. 4 (top panel), shows a broad bump near the critical temperature, a feature that is qualitatively consistent with the experiment (Fig. 3a). However, in stark contrast to the experimental data, our simulations find a positive and significant $X_{2}$ only in the vicinity of $T_{\mathrm{c}}$ (Fig. 4 -middle panel). Our finding of a vanishing $X_{2}$ at very low temperatures in the zig-zag phase is, in fact, consistent with an emergent timereversal symmetry of the low $T$-ordered state. To see this emergent symmetry, we first note that although there are three equivalent propagation directions for the zig-zag order, it has been shown experimentally and theoretically that the ground state of $a-\mathrm{RuCl}_{3}$ is a single- $Q$ zig-zag. Spins in such single- $Q$ zig-zag are collinear, with opposite spins sitting on alternating zig-zag chains of the honeycomb lattice. The total magnetization of such collinear bipartite antiferromagnetic order is the sum of the two sublattices: $\mathbf{M}=\left(M_{A}+M_{B}\right) \hat{\mathbf{n}}$, where $\hat{\mathbf{n}}$ is the collinear spin direction, and $\left\langle M_{B}\right\rangle=-\left\langle M_{A}\right\rangle$. Their contributions to the secondorder magnetic susceptibility $d$

$X_{2} \sim\left\langle M^{3}\right\rangle=\left\langle M_{A}^{3}\right\rangle+\left\langle M_{B}^{3}\right\rangle+3\left\langle M_{A}^{2} M_{B}\right\rangle+3\left\langle M_{A} M_{B}^{2}\right\rangle$

cancel each other due to the sublattice symmetry $\left\langle M_{A}^{3}\right\rangle=-\left\langle M_{B}^{3}\right\rangle$ and $\left\langle M_{A}^{2} M_{B}\right\rangle=-\left\langle M_{B} M_{A}^{2}\right\rangle$. The persistence of the quadratic $X_{2}$ 

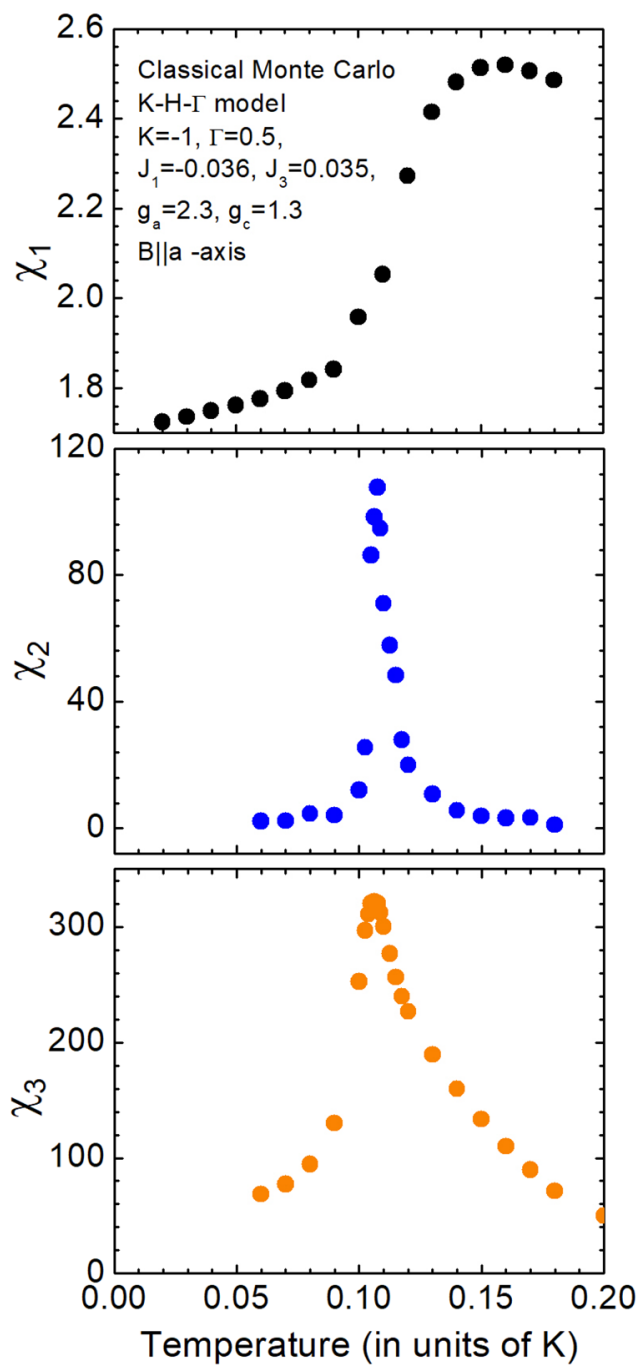

Fig. 4 CMC simulations. This figure shows the temperature dependence of the linear and nonlinear susceptibilities obtained in Monte Carlo calculations, for $B \| a$-axis. $X_{2}$ (middle panel) rises rapidly to positive values at $T_{C}$ reaches a maximum and drops sharply again below $T_{\mathrm{C}}$. This latter feature is in sharp contrast to the experimental results where the large positive $x_{2}$ persists to the lowest temperatures measured. Similar results in contrast to the experiment are obtained for the behavior of $X_{3}$. It also attains a positive value below $T \approx 0.11$ (in units of $K_{1}$ ), reaches a peak value near $T_{N}$, and rapidly decreases at lower temperatures (lower panel). Note that $y$-axis scale is arbitrary.

down to the lowest temperatures in our experiments thus highlights the unusual nature of the zig-zag order. This intriguing discrepancy could be due to nontrivial quantum fluctuations in the ordered state. Another possible scenario is that the nonzero $X_{2}$ results from a highly inhomogeneous multidomain zig-zag order at low temperatures. Indeed, at the interfaces between different zig-zag domains, the two sublattice collinear spins argument given above no longer applies and a nonzero $\chi_{2}$ could result from the complex noncollinear structure at the domain boundaries. Our experiments also imply that the low-temperature $X_{2}$ vanishes at the critical field $B^{*} \approx 2 \mathrm{~T}$. Interestingly, this critical field $B^{*}$ has recently been attributed to the so-called $Q$-flop transition identified both in neutron diffraction ${ }^{12,21}$ and terahertz spectroscopy ${ }^{41,42}$. Since the presence of the magnetic field in the $a b$ plane breaks the equivalence of the three zig-zag orientations, the $Q$-flop transition describes a repopulation of zig-zag domains in which two energetically unfavorable zig-zag domains are replaced by the third one. Such a realignment of the zig-zag domains also significantly reduces the noncollinear spins residing on the interfaces of different zig-zag order, thus giving rise to a reduced $X_{2}$. Detailed numerical simulations of the $Q$-flop transition and its effects on $X_{2}$ will be left for a further computational study. The breaking of the sublattice symmetry, which leads to a nonzero $X_{2}$ in the ground state, could also come at the dynamical level, for example, due to nontrivial quantum fluctuations with noncollinear high-order spin correlations in the ordered state $^{27}$. Other possibilities such as a stacking of the single- $Q$ collinear zig-zag order along the $c$-direction that breaks the sublattice symmetry cannot be ruled out either. Compared with CMC simulations another intriguing result from our experiments is the persistence of large $X_{3}$ in both high- and low-temperature regimes. The values of $X_{3}$ from CMC simulations approach zero very rapidly above $T_{\mathrm{c}}$ in contrast to the experimental large and positive values that persist for temperatures significantly greater than $T_{\mathrm{c}}$. The lowtemperature discrepancy of $X_{3}$ can be attributed to the absence of quantum fluctuations in CMC.

However, the importance of such fluctuations is borne out in similar calculations utilizing quantum chemistry methods discussed below. They are also seen in quantum Monte Carlo simulations in the pure Kitaev limit by Kamiya et al. ${ }^{43}$, which show a persistent positive $X_{3}$ down to the lowest temperatures. In quantum chemistry methods, we use ED of a closely related Hamiltonian employed in ref. ${ }^{38}$, which yields accurate results for the magnetization isotherms in good agreement with experimental results at the high field end. The calculated magnetization isotherms in this approach plotted in a manner similar to experiments are well fit with a single straight line (see Fig. S9), implying that only $x_{3}$ contributes. The values of $x_{3}$ extracted from such calculations are large and positive even at $T=0$ (Fig. 5). Also shown in Fig. 5 is the behavior of the calculated derivative $\mathrm{d}^{2} \mathrm{M} / \mathrm{d} B^{2}$, which displays a zero intercept for all temperatures. This implies that the dual-slope response, which we attribute to the presence of the complex multidomain zig-zag order or other sublattice-symmetry breaking mechanisms, is not found in the ED calculations. It is worth noting that the nonzero value of $X_{3}$ for $T \rightarrow 0$ is also found in the pure Kitaev model with quantum Monte Carlo calculations ${ }^{43}$. In such calculations, it is possible to secure a crossover of $X_{3}$ at a higher temperature; however, only through the antiferromagnetic Kitaev interaction. Many calculations ${ }^{32}$ rule out an antiferromagnetic scenario, but our experimental results suggest not to exclude this possibility.

Moving forward, any viable model for $a-\mathrm{RuCl}_{3}$ must explain the quadratic contribution to the magnetization in the antiferromagnetic zig-zag phase evident in our experiments. Further, it also has to account for the persistence of the large positive values of the third-order susceptibility for temperatures well above $T_{\mathrm{c}}$. It might be that the details of the material parameters will decide the magnitude and the temperature range over which a positive nonlinearity is stretched.

In summary, we have presented measurements of the nonlinear susceptibility in the Kitaev magnet $a-\mathrm{RuCl}_{3}$. Most significantly, our work has uncovered an anomalous quadratic response of the magnetization to a field that yields a large positive $X_{2}$ in the ordered state as $T \rightarrow 0$. This behavior is absent when $B \| c$-axis, suggesting a strong $2 \mathrm{D}$ nature of the order parameter. This anisotropy as well as the measured anisotropy of $X_{3}$ both above and below $T_{c}$ can serve as future characterization tools for pinning down specific models for proximate Kitaev materials. In addition, our observation of extended positive behavior of $X_{3}$ up to $50 \mathrm{~K}$ is consistent with previous circumstantial evidence that the Kitaevtype behavior with associated excitations persists up to fairly large temperatures $T \approx 60 \mathrm{~K} \gg T_{\mathrm{c}}^{6,10}$. The low field crossover, that is, the anomalous response with a quadratic term at low fields does not have to be confined to $a-\mathrm{RuCl}_{3}$ and the generality of its presence 

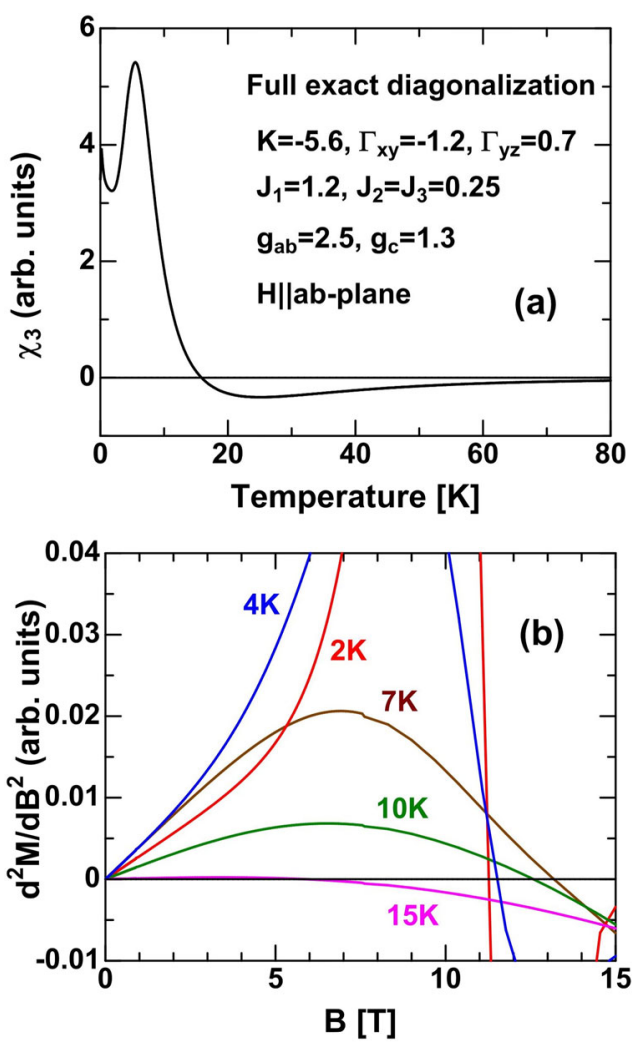

Fig. 5 Exact diagonalization calculations. This figure shows the nonlinear susceptibility $X_{3}$ in calculations employing exact diagonalization for $B$ in the $a b$-plane. The results obtained are identical whether $B \| a$-axis or $B \perp a$-axis. $X_{3}$ (panel (a)) rises rapidly to positive values at $T_{N}$ reaches a maximum and saturates to a large positive value at $T=0$. However, the zero-crossing occurs within $2 T_{N}$, whereas the experimental zero-crossing occurs at a much higher temperature. Panel (b) shows the second derivative, $d^{2} M / d B^{2}$, at various temperatures. This derivative passes zero at all temperatures-thus implying the absence of a $B^{2}$ term in the magnetization contrary to experimental findings.

in Kitaev or other spin liquid compounds should be established. Furthermore, our results could place constraints on models that attempt to explain experimental observations in $a-\mathrm{RuCl}_{3}$ and similar compounds outside the realm of Kitaev physics ${ }^{35,44}$. Is the anomalous $X_{2}$ and the extended positive $X_{3}$ a natural consequence of Kitaev- type models when quantum fluctuations are correctly accounted for or is it a peculiarity of $a-\mathrm{RuCl}_{3}{ }^{45}$ ? We note that the conditions to approach pure Kitaev without the nuisance of magnetic order experimentally are fairly easy to reach-the zig-zag order is destroyed in $a-\mathrm{RuCl}_{3}$ at relatively low pressures of $\approx 1 \mathrm{GPa}^{46,47}$. Equally important to carry this work forward will be attempts to predict an in-plane anisotropy of the nonlinear response in a quantitative manner, followed by further detailed experimental work in this regard.

\section{METHODS}

\section{Materials and experiments}

Our measurements were performed on high-quality single crystals similar to those used for recent linear magnetometry measurements ${ }^{13}$. A Quantum Design Magnetic Property Measurement System SQUID magnetometer capable of reaching $5 \mathrm{~T}$ was employed to obtain magnetization isotherms in the temperature range $2-300 \mathrm{~K}$.

\section{Simulations}

CMC as well as quantum chemistry-based ED calculations of the linear and nonlinear magnetic response in the $K-H-\Gamma$ mode $^{32}$ were performed using available packages on a supercomputer.

\section{DATA AVAILABILITY}

The datasets generated during and/or analyzed during the current study are available from the corresponding author on reasonable request.

Received: 24 January 2021; Accepted: 8 June 2021; Published online: 09 July 2021

\section{REFERENCES}

1. Kitaev, A. Anyons in an exactly solved model and beyond. Ann. Phys. 321, 2-111 (2006).

2. Jackeli, G. \& Khaliullin, G. Mott insulators in the strong spin-orbit coupling limit: from Heisenberg to a quantum compass and Kitaev models. Phys. Rev. Lett. 102, 017205 (2009).

3. Hermanns, J. K. M., Kimchi, I. \& Knolle, J. Physics of the Kitaev model: fractionalization, dynamic correlations, and material connections. Annu. Rev. Condens. Matter Phys. 9, 17-33 (2018).

4. Savary, L. \& Balents, L. Quantum spin liquids: a review. Rep. Prog. Phys. 80, 016502 (2017).

5. Kasahara, Y. et al. Majorana quantization and half-integer thermal quantum Hall effect in a Kitaev spin liquid. Nature 559, 227-231 (2018).

6. Do, S.-H. et al. Majorana fermions in the Kitaev quantum spin system $a-\mathrm{RuCl}_{3}$. Nat. Phys. 13, 1079-1084 (2017).

7. Wellm, C. et al. Signatures of low-energy fractionalized excitations in $a-\mathrm{RuCl}_{3}$ from field-dependent microwave absorption. Phys. Rev. B 98, 184408 (2018).

8. Banerjee, A. et al. Proximate Kitaev quantum spin liquid behaviour in a honeycomb magnet. Nat. Mater. 15, 733-740 (2016).

9. Banerjee, A. et al. Neutron scattering in the proximate quantum spin liquid $a-$ $\mathrm{RuCl}_{3}$. Science 356, 1055-1059 (2017).

10. Sandilands, L. J., Tian, Y., Plumb, K. W., Kim, Y.-J. \& Burch, K. S. Scattering continuum and possible fractionalized excitations in $a-\mathrm{RuCl}_{3}$. Phys. Rev. Lett. 114, 147201 (2015)

11. Wang, Y. et al. The range of non-Kitaev terms and fractional particles in $a-\mathrm{RuCl}_{3}$. npj Quantum Mater. 5, 14 (2020).

12. Sears, J. A. et al. Magnetic order in $a-\mathrm{RuCl}_{3}$ : a honeycomb-lattice quantum magnet with strong spin-orbit coupling. Phys. Rev. B 91, 144420 (2015).

13. Lampen-Kelley, P. et al. Anisotropic susceptibilities in the honeycomb Kitaev system $a-\mathrm{RuCl}_{3}$. Phys. Rev. B 98, 100403(R) (2018).

14. Narita, N. \& Yamada, I. Nonlinear magnetic-susceptibility of two-dimensional magnets $\left(\mathrm{C}_{n} \mathrm{H}_{2 n .1} \mathrm{NH}_{3}\right)_{2} \mathrm{CuCl}_{4}$ with $n=1,2$ and 3. J. Phys. Soc. Jpn. 65, 4054 (1996).

15. Bitla, Y. \& Kaul, S. N. Mean-field treatment of nonlinear susceptibilities for a ferromagnet of arbitrary spin. Europhys. Lett. 96, 37012 (2011).

16. Morin, P. \& Schmitt, D. Third-order magnetic susceptibility as a new method for studying quadrupolar interactions in rare-earth compounds. Phys. Rev. B 23, 5936-5949 (1981)

17. Gingras, M. J. P. et al. Static critical behavior of the spin-freezing transition in the geometrically frustrated pyrochlore antiferromagnet $\mathrm{Y}_{2} \mathrm{Mo}_{2} \mathrm{O}_{7}$. Phys. Rev. Lett. 78, 947-950 (1997).

18. Ramirez, A. P., Espinosa, G. P. \& Cooper, A. S. Strong frustration and dilutionenhanced order in a quasi-2D spin glass. Phys. Rev. Lett. 64, 2070-2073 (1990).

19. Suzuki, M. Phenomenological theory of spin-glasses and some rigorous results. Prog. Theor. Phys. 58, 1151-1165 (1977).

20. Lai, L.-Q., Li, Z., Yu, Y.-B. \& Liu, Q.-H. Third-order magnetic susceptibility of an ideal Fermi gas. Commun. Theor. Phys. 70, 619 (2018).

21. Banerjee, A. et al. Excitations in the field-induced quantum spin liquid state of $a$ $\mathrm{RuCl}_{3}$. npj Quantum Mater. 3, 8 (2018).

22. Kubota, Y., Tanaka, H., Ono, T., Narumi, Y. \& Kindo, K. Successive magnetic phase transitions in $a-\mathrm{RuCl}_{3}: \mathrm{XY}$-like frustrated magnet on the honeycomb lattice. Phys. Rev. B 91, 094422 (2015).

23. Shivaram, B. S., Hinks, D. G., Kumar, P., Andrade, M. \& Maple, M. B. Universality in the magnetic response of metamagnetic metals. Phys. Rev. B 89, 241107(R) (2014).

24. Kushauer, J. \& Kleemann, W. Critical behaviour of the linear and non-linear magnetic susceptibilities of $\mathrm{FeCl}_{2}$. J. Phys. 7, L1-L6 (1995). 
25. Fujiki, S. \& Katsura, S. Nonlinear susceptibility in the spin glass. Prog. Theor. Phys. 64, 1130 (1981).

26. Schiffer, P., Ramirez, A., Franklin, K. N. \& Cheong, S. W. Interaction-induced spin coplanarity in a Kagomé magnet: $\mathrm{SrCr}_{9 p} \mathrm{Ga}_{12-9 p} \mathrm{O}_{19}$. Phys. Rev. Lett. 77, 2085-2088 (1995).

27. Ramirez, A. P. et al. Nonlinear susceptibility as a probe of tensor spin order in $\mathrm{URu}_{2} \mathrm{Si}_{2}$. Phys. Rev. Lett. 68, 2680-2683 (1992).

28. Kitagawa, J. et al. Third-order magnetic susceptibility and quadrupolar order parameter of Kondo-lattice compound $\mathrm{Ce}_{3} \mathrm{Pd}_{2} \mathrm{OGe}_{6}$. J. Phys. Soc. Jpn. 69, 883-887 (2007).

29. Ramirez, A. P. et al. Nonlinear susceptibility: a direct test of the quadrupolar Kondo effect in $\mathrm{UBe}_{13}$. Phys. Rev. Lett. 73, 3018-3021 (1994).

30. Bauer, E. D. et al. Nonlinear susceptibility: evidence for antiferroquadrupolar fluctuations and a nonmagnetic $\Gamma_{1}$ ground state in the heavy fermion superconductor $\mathrm{PrOs}_{4} \mathrm{Sb}_{12}$. Phys. Rev. B73, 094511 (2006).

31. Zheludev, A. et al. Field-induced commensurate-incommensurate phase transition in a Dzyaloshinskii-Moriya spiral antiferromagnet. Phys. Rev. Lett. 78 4857-4860 (1997).

32. Winter, S. M., Riedl, K., Kaib, D., Coldea, R. \& Valenti, R. Probing a $-\mathrm{RuCl}_{3}$ beyond magnetic order: effects of temperature and magnetic field. Phys. Rev. Lett. 120, 077203 (2018).

33. Winter, S. M. et al. Models and materials for generalized Kitaev magnetism. J. Phys. 29, 493002 (2017).

34. Janssen, L., Andrade, E. C. \& Vojta, M. Magnetization processes of zigzag states on the honeycomb lattice: identifying spin models for $a-\mathrm{RuCl}_{3}$ and $\mathrm{Na}_{2} \mathrm{IO}_{3}$. Phys. Rev. B 96, 064430 (2017).

35. Zhou, Y., Li, Y. D., Yang, X. \& Chen, G. Non-Kitaev spin liquids in Kitaev materials. Phys. Rev. B 99, 205119 (2019).

36. Wilson, E. \& Haraldsen, J. Understanding the magnetic interactions of the zig-zag honeycomb lattice: application to $\mathrm{RuCl}_{3}$. https://meetings.aps.org/Meeting/ MAR21/Session/E39.11 (2021).

37. Kim, Y.-J. Ferromagnetic Kitaev interactions and magnetic anisotropy in $a-\mathrm{RuCl}_{3}$. https://meetings.aps.org/Meeting/MAR21/Session/A54.1 (2021).

38. Yadav, R. et al. Kitaev exchange and field-induced quantum spin-liquid states in honeycomb $a-\mathrm{RuCl}_{3}$. Sci. Rep. 6, 37925 (2016).

39. Winter, S. M. et al. Breakdown of magnons in a strongly spin-orbital coupled magnet. Nat. Commun. 8, 1152 (2017).

40. Kim, H.-S. \& Kee, H.-Y. Crystal structure and magnetism in $a-\mathrm{RuCl}_{3}:$ An ab initio study. Phys. Rev. B 93, 155143 (2016).

41. $\mathrm{Wu}$, L. et al. Field evolution of magnons in $a-\mathrm{RuCl}_{3}$ by high-resolution polarized terahertz spectroscopy. Phys. Rev. B 98, 094425 (2018).

42. Ozel, I. O. et al. Magnetic field-dependent low-energy magnon dynamics in $a$ $\mathrm{RuCl}_{3}$. Phys. Rev. B 100, 085108 (2019).

43. Kamiya, Y., Yoshitake, J., Kato, Y., Nasu, J. \& Motome, Y. Nonlinear magnetic susceptibility in the Kitaev model. https://meetings.aps.org/Meeting/MAR19/ Session/A37.3 (2019).

44. Modic, K. A. et al. Scale-invariance of a spin liquid in high magnetic fields. Nat. Phys. 17, 240-244 (2021).

45. Dai, Z. et al. Crystal structure reconstruction in the surface monolayer of the quantum spin liquid candidate $a-\mathrm{RuCl}_{3} .2 \mathrm{D}$ Mater. 7, 035004 (2020).

46. Cui, Y. et al. High-pressure magnetization and NMR studies of $a-\mathrm{RuCl}_{3}$. Phys. Rev. $B$ 96, 205147 (2017).

47. Wang, Z. et al. Pressure-induced melting of magnetic order and emergence of a new quantum state in $a-\mathrm{RuCl}_{3}$. Phys. Rev. B 97, 245149 (2018).

\section{ACKNOWLEDGEMENTS}

We acknowledge many useful conversations with and suggestions for experimental work from Steve Nagler, David Mandrus, and Arnab Banerjee. We are grateful to Jiaqiang Yan for the sample of single-crystal $a-\mathrm{RuCl}_{3}$. We acknowledge useful discussions and correspondence with Pradeep Kumar, Yoshitomo Kamiya, Joji Nasu, Nandini Trivedi, Collin Broholm, and Mike Zhitomirsky. S.N. thanks U. Nitzsche for technical assistance. Z.F. and G.-W.C. are partially supported by the Center for Materials Theory as a part of the Computational Materials Science (CMS) program, funded by the US Department of Energy, Office of Science, Basic Energy Sciences, Materials Sciences, and Engineering Division. This work was also partially supported by ONR under the Naval Research Laboratory base program. The work of B.S.S. was supported by NSF Award DMR2016909 and in. Germany was supported by DFG through SFB 1143 project A05.

\section{AUTHOR CONTRIBUTIONS}

L.H. and J.P. collected the data. L.H. and B.S.S. analyzed the data and made the plots. Z.F. and G.-W.C. performed the classical Monte Carlo simulations. S.N. and J.v.d.B. calculated the magnetization through exact diagonalization of the Hamiltonian. M.O. B.S.S., and G.-W.C. planned the research and developed the manuscript, which was edited by all authors.

\section{COMPETING INTERESTS}

The authors declare no competing interests.

\section{ADDITIONAL INFORMATION}

Supplementary information The online version contains supplementary material available at https://doi.org/10.1038/s41535-021-00364-z.

Correspondence and requests for materials should be addressed to B.S.S.

Reprints and permission information is available at http://www.nature.com/ reprints

Publisher's note Springer Nature remains neutral with regard to jurisdictional claims in published maps and institutional affiliations.

Open Access This article is licensed under a Creative Commons cc Attribution 4.0 International License, which permits use, sharing, adaptation, distribution and reproduction in any medium or format, as long as you give appropriate credit to the original author(s) and the source, provide a link to the Creative Commons license, and indicate if changes were made. The images or other third party material in this article are included in the article's Creative Commons license, unless indicated otherwise in a credit line to the material. If material is not included in the article's Creative Commons license and your intended use is not permitted by statutory regulation or exceeds the permitted use, you will need to obtain permission directly from the copyright holder. To view a copy of this license, visit http://creativecommons. org/licenses/by/4.0/.

(c) The Author(s) 2021 